\title{
Intérêt de la photothérapie au laser pour le traitement des mucites de la cavité buccale. Présentation d'un cas et revue de la littérature
}

\author{
Géraldine Lescaille ${ }^{1}$, Philippe Lang ${ }^{2}$, Didier Ernenwein ${ }^{3}$, Marie-José Javelot ${ }^{1}$, \\ Vianney Descroix ${ }^{1, \star}$ \\ 1 Service d'Odontologie, Groupe hospitalier Pitié-Salpêtrière, Paris, France \\ 2 Service de Radiothérapie, Groupe hospitalier Pitié-Salpêtrière, Paris, France \\ 3 Service de Chirurgie maxillo-faciale, Groupe hospitalier Pitié-Salpêtrière, Paris, France
}

(Reçu le 14 mai 2010, accepté le 23 mai 2010)

Mots clés :

mucite buccale /

laser /

douleur /

cancers des voies

aéro-digestives

\begin{abstract}
Résumé - La mucite est une complication classique de la radiothérapie et/ou de la chimiothérapie dans le traitement des cancers des voies aéro-digestives supérieures. Sa prise en charge est avant tout symptomatique : prescription d'un traitement antalgique, topique et systémique. Depuis quelques années, l'utilisation de différents lasers s'est révélée particulièrement intéressante pour le traitement préventif et curatif des mucites radio et/ou chimio-induites.

L'objectif de cet article est de faire le point sur les mécanismes d'action du laser de basse énergie et ses indications dans le traitement de lésions muqueuses spécifiques. Nous rapportons ici l'exemple d'un cas de mucite de grade 3 radio-induite chez un patient en cours de radiothérapie postopératoire pour un carcinome épidermoïde du rebord maxillaire. La prise en charge classique (soins locaux et antalgiques) a été mise en place, en association avec des séances de laser Nd :YAP $(1,34 \mu \mathrm{m})$. Le retour à une toxicité muqueuse $\leq 2$ et une cicatrisation de la plupart des zones ulcérées ont été observés dès la première semaine d'application. Parallèlement, un effet antalgique rapide était constaté avec une diminution de la douleur qui est passée de 90 à 30 sur l'EVA.
\end{abstract}

\begin{abstract}
Lasers in the treatment of oral mucositis. Report of a case and review of litterature. The mucositis is a common complication of radiotherapy and/or chemotherapy in the treatment of head and neck cancers. Its management is symptomatic with topical and systemic analgesics. In recent years, the use of various lasers has proved particularly interesting for the preventive and curative treatment of mucositis induced by radiotherapy and chemotherapy.

The aim of this article is to review the mechanisms of action of low energy laser and its indications in the treatment of mucosal lesion. We report here the case of mucositis grade 3 radiation-induced in a patient during postoperative radiotherapy for squamous cell carcinoma of the maxillary. Classical treatment (local care and analgesics) was set up in conjunction with sessions of $\mathrm{Nd}$ :YAP $(1.34 \mathrm{~mm})$. The return to $\leq 2$ mucosal toxicity and scarring of the ulcerated areas were most observed in the first week of application. Meanwhile a rapid analgesic effect was observed with a decrease in pain that went from 90-30 on the VAS.
\end{abstract}

La radiothérapie, exclusive ou postopératoire, occupe une place fondamentale dans le traitement des cancers des voies aéro-digestives supérieures. Dans les formes avancées, le bénéfice d'une chimiothérapie concomitante à l'irradiation a été clairement démontré : elle permet d'améliorer le contrôle local

\footnotetext{
^Correspondance : vianney.descroix@psl.aphp.fr
}

des tumeurs et la survie globale des patients [1]. Cette intensification thérapeutique s'accompagne d'une augmentation significative de la toxicité aiguë, en particulier d'une radiomucite de grade 3, chez 40 à $60 \%$ des patients [2-4]. Son impact est majeur en termes de morbidité liée au traitement : le recours aux antalgiques de palier 3, à l'hospitalisation et au support nutritionnel par sonde gastrique intéressent 
respectivement $60 \%, 30 \%$ et $40 \%$ des patients. La compliance est le plus souvent sous-optimale et, dans de rares cas, l'irradiation doit être suspendue. Sur le plan clinique, la mucite se présente d'abord sous la forme d'un érythème simple (grade 1), puis d'érosions et d'ulcérations profondes, éparses (grade 2) ou confluentes (grade 3), siégeant entre autres sur la muqueuse non kératinisée (joues, langue, voile, lèvres). La radiomucite de grade $\geq 2$ survient généralement après la $3^{\text {e }}$ semaine de traitement et cicatrise en 4 à 6 semaines après la fin du traitement. Elle se caractérise par une sensation de brûlures et d'une douleur angineuse augmentant progressivement, ayant un retentissement direct sur la nutrition et la courbe de poids. Ces lésions résultent d'une toxicité directe des traitements de chimiothérapie et de radiothérapie sur la muqueuse buccale, mais également d'un processus indirect dû à la surinfection qui potentialise la sévérité des lésions initiales $[5,6]$.

Le traitement de la mucite est avant tout symptomatique associant soins locaux et antalgiques. Les bains de bouche à base de bicarbonate à $14 \%$ constituent le traitement topique de référence, bien qu'il ne soit pas spécifique. Les anti-inflammatoires stéroïdiens sont fréquemment utilisés en cure courte $(\leq 7 \mathrm{j})$ en fin d'irradiation afin d'éviter l'arrêt du traitement; les antibiotiques et les antifongiques n'étant indiqués qu'en cas de surinfection évidente. Différents traitements préventifs et curatifs ont fait l'objet de nombreuses études mais peu se sont révélés efficaces [3]. Depuis quelques années, l'utilisation de la photothérapie au laser s'est montrée intéressante : elle a fait l'objet de nombreuses publications et d'une attention particulière du groupe Mucites MASCC/ISOO (Multinational Association of Supportive Care in Cancer/International Society for Oral Oncology) [7].

L'efficacité de ce traitement est illustrée par la présentation du cas d'un patient traité pour un carcinome épidermoïde buccal présentant une mucite de grade 3 après 3 semaines et demie de traitement par radiothérapie externe (35 Gy). La prise en charge de la mucite a consisté en l'administration d'antalgiques par voies topique et systémique en association à des séances de photothérapie avec le laser $\mathrm{Nd}$ :Yap.

\section{Observation}

Il s'agit d'un patient de 83 ans, non fumeur, sans antécédents médico-chirurgicaux notables, atteint d'un carcinome épidermoïde moyennement différencié du maxillaire gauche, classé cT4N2bM0. La prise en charge oncologique a été réalisée sur le groupe hospitalier Pitié-Salpêtrière en collaboration avec les services de Chirurgie maxillo-faciale, d'0ncoradiothérapie et d'Odontologie. Après bilan bucco-dentaire, une maxillectomie partielle gauche associée à un curage cervical des aires ganglionnaires 1 à 5 homolatérales a été réalisée ; les avulsions dentaires ont été effectuées dans le même temps chirurgical. Une prothèse obturatrice, adaptée en peropératoire, a été mise en place. Des soins prothétiques ont été entrepris en postopératoire pendant le traitement de radiothérapie.
La radiothérapie postopératoire a débuté 8 semaines après l'intervention. Le plan de traitement prévoyait une dose prophylactique de 45-50 Gy sur la zone d'exérèse chirurgicale et 66 Gy sur la région cervicale gauche en raison de l'envahissement ganglionnaire massif avec rupture capsulaire. L'irradiation a été réalisée, après repérage scannographique et dosimétrie conformationnelle préservant la glande parotide controlatérale, avec un fractionnement standard (2 Gy par fraction, $10 \mathrm{~Gy}$ par semaine). À la dose de $35 \mathrm{~Gy}$, l'irradiation a été suspendue pour une durée de 7 jours à la demande du radiothérapeute en raison d'une mucite de grade 3 intéressant la commissure labiale, la face interne de la joue et le bord latéral de langue (Fig. 1). Les ulcérations buccales étaient multiples, associées à une épidermite et un œdème cervical. La douleur était alors évaluée à 90 sur l'EVA.

\section{Traitement}

Le traitement antalgique comprenait du paracétamol (1 g toutes les $6 \mathrm{~h}$ ) et du néfopam (Acupan ${ }^{\circledR}$ ) en cas de douleurs brutales. Un traitement topique avec une préparation magistrale associant de la xylocaïne $2 \%$ (2 flacons), de la chlorhexidine $0,5 \%$ (Eludril ${ }^{\circledR} 90 \mathrm{~mL} 1$ flacon) de la nystatine (Mycostatine ${ }^{\circledR}$ ) buvable $(24 \mathrm{~mL}$ ) et du bicarbonate de sodium à $14 \%$ (400 mL) a été prescrit, ainsi que du sérum physiologique pour les fosses nasales.

Deux séances de laser $\mathrm{Nd}$ :YAP $(1,34 \mu \mathrm{m})$ à trois jours d'intervalle ont été réalisées. Le laser a été appliqué à $1 \mathrm{~cm}$ de la muqueuse, en trois stimulations, à une puissance de 5 watts, sur toute la surface de la muqueuse buccale.

\section{Résultats}

Des photographies ont été réalisées avant la première séance de laser (Fig. 1) et une semaine plus tard (Fig. 2). La douleur a été mesurée sur une EVA avant le début du traitement (antalgiques, antiseptiques et deux séances de laser) et une semaine après le début du traitement. Une diminution de l'intensité douloureuse (EVA de 90 à 30) a été observée ; après une semaine, une diminution significative de la superficie des lésions, avec une régression complète sur la lèvre inférieure, une nette régression des lésions linguale et palatine qui étaient passées du grade 3 au grade 1 .

\section{Commentaires}

La toxicité muqueuse constitue le facteur limitant du traitement non chirurgical des cancers des VADS, notamment en cas d'association radio-chimiothérapie. Concernant la radiothérapie, les modalités de traitement auraient une incidence sur leur développement. En effet, l'accélération (même dose totale sur un temps plus court), l'hyperfractionnement (plusieurs fractions par jour) et la dose totale élevée (80 Gy) augmentent significativement la toxicité muqueuse, donnant 50$60 \%$ de mucite de grade 3 . L'irradiation conformationnelle, 

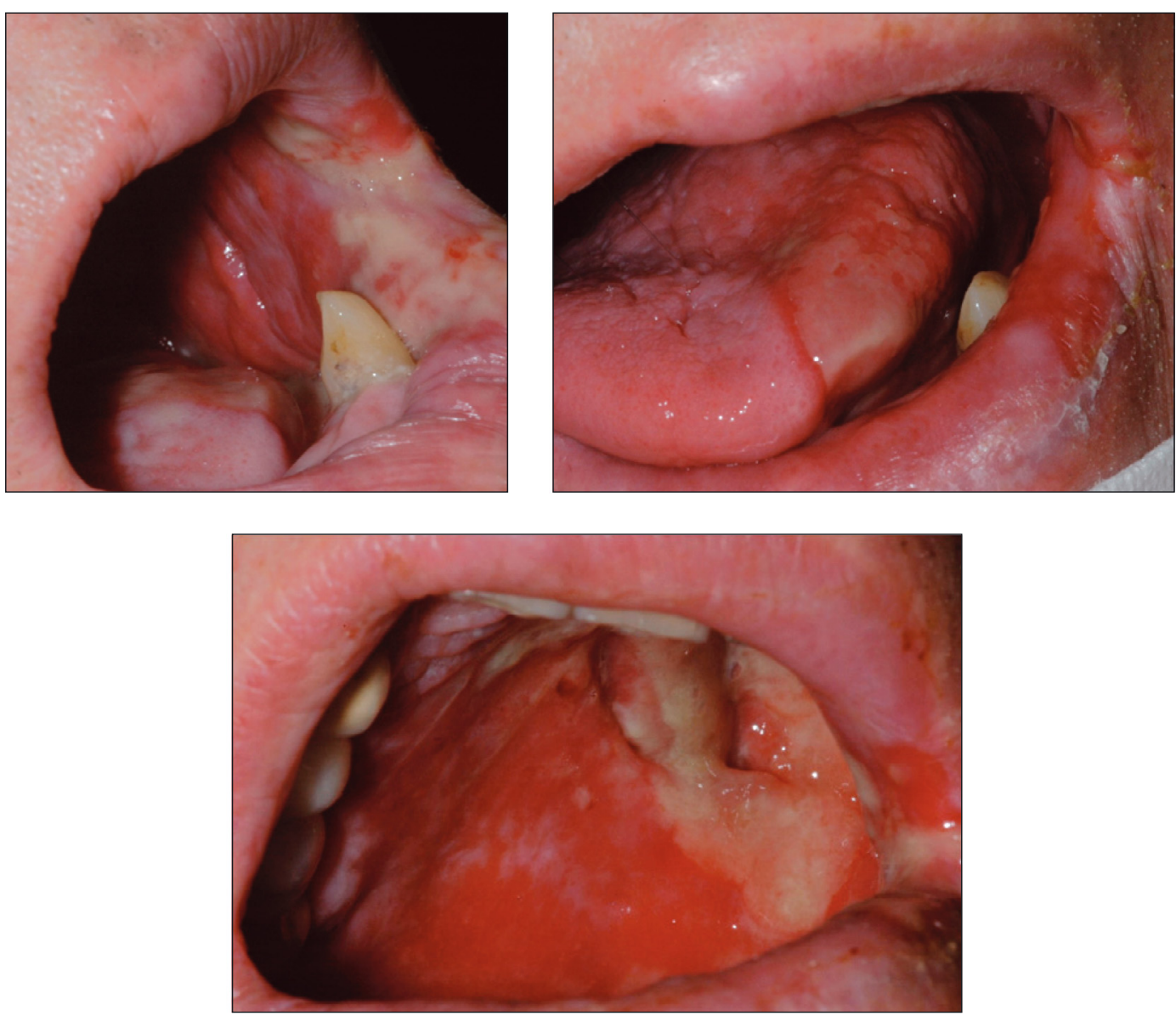

Fig. 1. Lésions de mucite grade 3 (langue, lèvre, joue et palais) chez un patient traité par radiothérapie externe après maxillectomie partielle. Fig. 1. Grade 3 mucositis lesions (tongue, lip, cheek and palate) in a patient treated with external radiotherapy after partial maxillectomy.

avec ou sans modulation d'intensité, éventuellement guidée par l'image, assure une meilleure définition des volumes cibles et permet par conséquent de réduire le volume des tissus sains irradiés à fortes doses [8]. Le traitement de la mucite est avant tout symptomatique, son objectif principal étant une diminution de l'intensité de la douleur. Ainsi, l'utilisation d'anesthésiques en application topique est souvent préconisée ainsi que l'emploi de médicaments anti-inflammatoires et antalgiques ou encore l'utilisation d'agents protecteurs de la muqueuse buccale, notamment la polyvinylpyrrolidone [7]. La prescription de pansements gastriques est également proposée pour les dysphagies : sel d'aluminium (sucralfate), antiacides ou argiles. Le traitement des mucites repose également sur la prévention des surinfections locales. Outre l'intérêt d'un bilan bucco-dentaire et d'une mise en état buccale préalable, le «bain de bouche magique » associant antifongique, antiseptique (chlorhexidine), bicarbonate de soude et anesthésique local (Xylocaïne ${ }^{\circledR}$ ) est très souvent prescrit mais reste de conception empirique; sa composition est controversée, notamment quant à l'intérêt de l'antifongique. Plus récemment, des thérapies comme le GMCSF (granulocyte macrophage colony stimulating factor) et l'amifostine (Ethyol ${ }^{\circledR}$ ) ont été proposées pour limiter la toxicité liée aux traitements de radio/chimiothérapie, notamment la mucite et la xérostomie. Ces derniers n'ont pas fait la preuve de leur efficacité et par conséquent leur utilisation n'a pas été retenue dans les consensus de l'ASCO 2008 pour le traitement standard des cancers des VADS. Le palifermin (Kepivance ${ }^{\circledR}$ ), une protéine analogue au facteur de croissance des kératinocytes naturels (KGF), obtenue par biotechnologie, a en revanche fourni des résultats intéressants dans la prise en charge des mucites sévères liées aux traitements de conditionnement réalisés avant les greffes de cellules souches hématopoïétiques. Ce produit injectable est en cours d'évaluation pour les mucites liées aux traitements des cancers buccaux [9].

L'intérêt de la photothérapie au laser dans le traitement préventif et curatif des mucites buccales chimio et/ou radioinduites a été mis en évidence dans de nombreuses études. Plusieurs études randomisées ont observé une diminution significative des mucites de grade 3 et 4 chez les patients traités par laser après chimiothérapie aplasiante, pendant le traitement de conditionnement avant les greffes de cellules souches hématopoïétiques dans le cadre du traitement des hémopathies malignes. Ainsi dès 1997, Cowen et al. avaient 

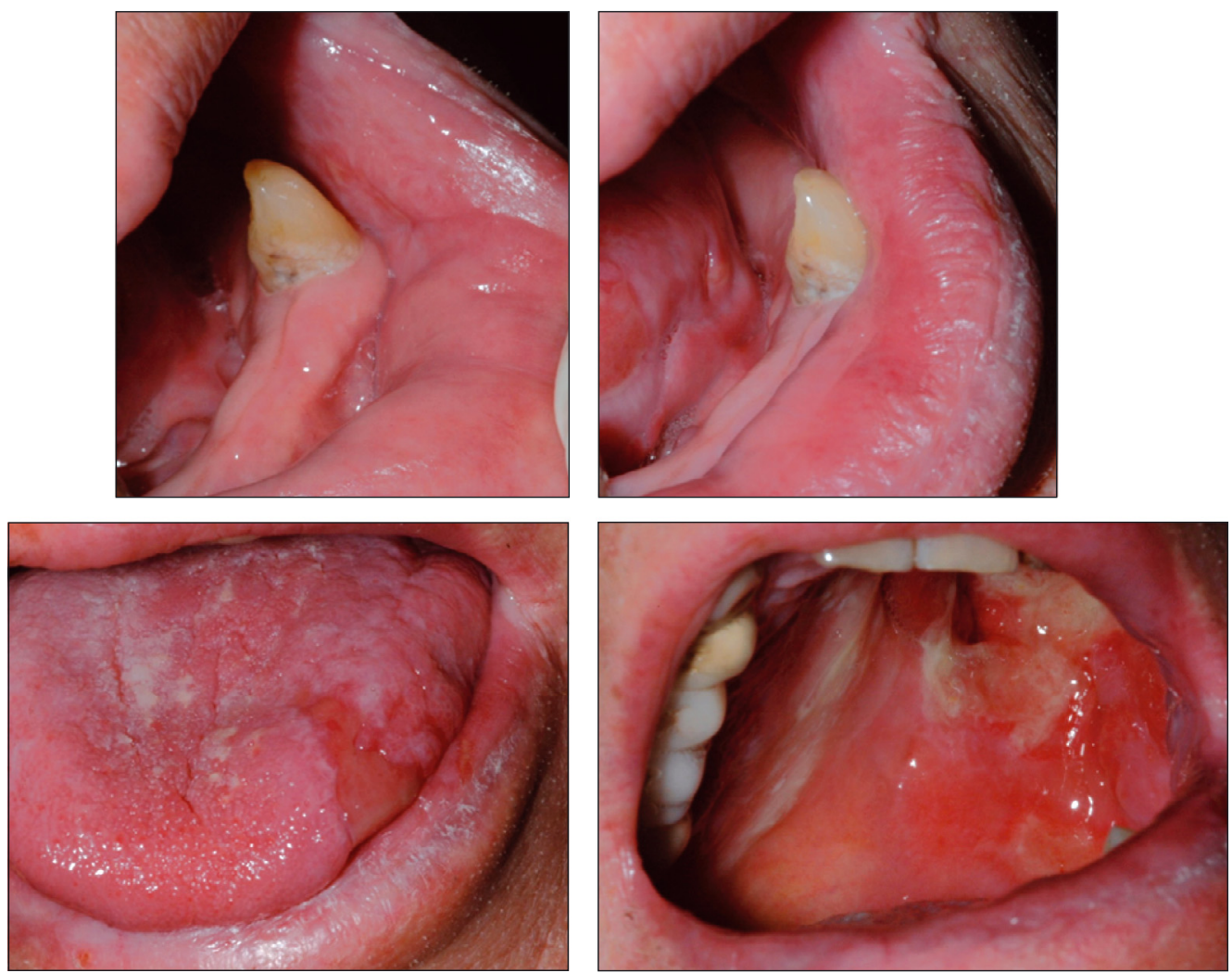

Fig. 2. Cicatrisation complète des lésions de mucite sur la lèvre et la joue, et nette régression des lésions linguales et palatines après 2 séances de photothérapie au laser $\mathrm{Nd}:$ Yap.

Fig. 2. Complete healing of mucositis lesions on the lip and cheek, and net regression of lingual and palatine lesions after 2 sessions of laser Nd:Yap phototherapy.

observé dans une étude randomisée en double aveugle une diminution significative de l'incidence $(p=0,01)$ et de la durée $(p=0,01)$ des mucites de grade 3 dans le groupe de patients traités par laser He-Ne $(632,8 \mathrm{~nm})$, par rapport au groupe contrôle [10]. En 2007, Antunes et al. et Schubert et al. ont observé, dans des études randomisées en double aveugle, les effets préventifs du laser diode $[11,12]$. Ainsi Antunes et al. rapportent que $94,7 \%$ des patients traités avec laser n'ont pas dépassé la mucite de grade 2, 63,2\% étant au grade 0 ou 1 (sans ulcérations), comparativement à $31,5 \%$ des patients pour le groupe contrôle $(P<0,001)$.

Par ailleurs, l'intérêt du laser de basse énergie a été démontré plus récemment dans le traitement préventif et curatif des mucites radio-induites chez des patients traités pour un cancer des VADS. Une première étude multicentrique randomisée, publiée en 1999 par Bensadoun et al., a étudié l'effet du laser He:Ne sur 30 patients. Conformément à ce qui a été décrit avec les mucites lors des greffes de cellules souches hématopoiétiques, l'incidence des mucites de grade 3 était de $7,6 \%$ dans le groupe laser contre $35,2 \%$ dans le groupe contrôle $(P<0,01)$ [13]. Par la suite, plusieurs types de lasers de basse énergie ont été évalués dans cette indication. Ainsi, Arora et al. (2008) ont étudié l'effet du laser He:Ne sur 24 patients traités par radio-chimiothérapie pour des cancers des VADS [14]. Simoes et al. (2009) ont comparé l'effet de deux types de lasers et de deux protocoles d'administration (1 fois/semaine et 3 fois/semaine) sur 39 patients, InGaAlP laser $(660 \mathrm{~nm}, 40 \mathrm{~mW} / \mathrm{cm})$ versus GaAlAs laser $(808 \mathrm{~nm}, 1 \mathrm{~W} / \mathrm{cm})$ [15]. Ils rapportent un meilleur contrôle de l'intensité de la douleur avec le laser de plus faible énergie avec un protocole de 3 fois/semaine. Zanin et al. (2009) ont obtenu des résultats similaires avec un laser diode $660 \mathrm{~nm}$ utilisé 2 fois/semaine, dans une étude portant sur 72 patients (groupe laser versus groupe contrôle $(n=36)$ ) [16].

Les lasers hélium-néon ( $\mathrm{He} / \mathrm{Ne}$, longueur d'ondes $632,8 \mathrm{~nm}$ ) et à diode (longueur d'ondes 650 à $905 \mathrm{~nm}$ ) sont les plus fréquemment rapportés dans ces indications, comme le laser InGaAIP de $660 \mathrm{~nm}$ [17] et $685 \mathrm{~nm}$ [18] et le laser GaAlAs de $830 \mathrm{~nm}$ [19]. Bien que l'intérêt du laser dans les mucites soit maintenant incontestable, la question de la puissance utilisée et du protocole fait actuellement débat. Il semble que les protocoles sur 2 à 3 jours/semaine soient plus efficaces que les protocoles proposant 1 utilisation/semaine. Simoes et al. ont obtenu de meilleurs résultats avec le laser de plus faible énergie [15]. Ces résultats restent à confirmer et des études supplémentaires devront préciser les paramètres suivant : le type de laser et sa longueur d'onde, la puissance et l'énergie administrée par $\mathrm{cm}^{2}$. 
Par ailleurs, bien que les effets antalgique, antiinflammatoire et cicatrisant des lasers de faible puissance soient décrits cliniquement depuis plusieurs années [20], la compréhension de leurs mécanismes d'action est récente et encore partielle. Quelques études permettent d'en expliquer les effets sur l'inflammation, la cicatrisation et la douleur dans les mucites radio- et/ou chimio-induites. Que ce soit au décours d'une chimiothérapie ou d'une radiothérapie, sur la muqueuse buccale ou gastro-intestinale, la production de radicaux libres (reactive oxygen species ou ROS) semble être l'événement le plus précoce, susceptible d'entraîner la formation des mucites [21]. Les lasers de basse énergie auraient une action directe sur le système énergétique mitochondrial, par diminution de la production de radicaux libres induits par les traitements anticancéreux et par activation de la transformation des fibroblastes en myofibroblastes. Par ailleurs, les ROS agissent d'une part directement sur la muqueuse et les vaisseaux et d'autre part indirectement en déclenchant une réaction inflammatoire locale par l'activation de facteurs de transcription, comme le $\mathrm{NF}_{\kappa} \mathrm{B}$ qui représente la voie centrale d'activation de nombreux gènes des médiateurs de la réaction inflammatoire (TNF $\alpha$, IL1 $\beta$ ou IL6) à l'origine de dommages tissulaires complexes survenant dans la muqueuse [21]. Des études, comme celle de Mafra de Lima (2008) [22], démontrent que le laser diode (GaAs) diminue l'expression des transcrits du TNF $\alpha$ via le $\mathrm{NF}_{\kappa} \mathrm{B}$, dans un modèle expérimental d'inflammation respiratoire induite par les lipopolysaccharides bactériens chez le rat. Il a été démontré que le laser diode de faible puissance à GaAs $(904 \mathrm{~nm})$ réduit de manière significative la migration cellulaire des polynucléaires neutrophiles dans un modèle expérimental de péritonite induit par les lipopolysaccharides bactériens [23]. Dans l'étude de Lim et al. (2007) [24], l'irradiation par le laser $635 \mathrm{~nm}$ a permis de mettre en évidence une inhibition du relarguage des prostaglandines inflammatoires (PGE2) et des deux isoformes (1 et 2) de la cyclooxygénase [25]. Il a été démontré que les lasers de faible puissance avaient par ailleurs une action antalgique en augmentant la synthèse et le relarguage des endorphines, ainsi qu'en diminuant le relarguage de récepteurs nociceptifs comme la bradykinine et la sérotonine [26-29].

Pour le potentiel cicatrisant, França et al. (2008) ont étudié l'aspect histologique des effets préventifs et thérapeutiques du laser diode (GaAs) dans des mucites chimio-induites chez le hamster : ils ont observé une augmentation de l'angiogenèse, du nombre de fibres de collagène, et de l'organisation du tissu de granulation dans les groupes traités avec le laser; et ce de manière plus importante. Ces effets sont plus marqués dans le groupe thérapeutique que dans le groupe préventif [30]. Il a été en outre montré in vitro un effet du laser de faible puissance sur la prolifération cellulaire [32].

\section{Conclusion}

La radio et/ou chimomucite constitue un véritable challenge thérapeutique en raison de sa morbidité aiguë. Malgré un suivi de plus en plus personnalisé pour le support nutritionnel et le confort du patient, la compliance des patients aux associations radio-chimiothérapie dans le cancer localement avancé des VADS n'excède pas $60 \%$ et demeure donc sous-optimale. Le laser de faible puissance semble capable de réduire la sévérité et l'incidence des mucites radioinduites et apparaît comme une technique efficace, simple et atraumatique. Toutefois, le groupe MASCC/ISO0 ne recommande ce traitement préventif que pour les institutions qui disposent déjà d'un laser de faible puissance et qui en ont l'expérience, car elle nécessite des moyens coûteux en temps et en personnel.

Conflit d'intérêt : aucun.

\section{Références}

1. Pignon JP, le Maître A, Maillard E, Bourhis J. MACH-NC Collaborative Group. Meta-analysis of chemotherapy in head and neck cancer (MACH-NC): an update on 93 randomised trials and 17,346 patients. Radiother Oncol 2009;92:4-14.

2. Trotti A, Bellm LA, Epstein JB, Frame D, Fuchs HJ, Gwede CK, Komaroff E, Nalysnyk L, Zilberberg MD. Mucositis incidence, severity and associated outcomes in patients with head and neck cancer receiving radiotherapy with or without chemotherapy: a systematic literature review. Radiother Oncol 2003;66:253-62.

3. Bourhis J, Lapeyre M, Tortochaux J, Rives M, Aghili M, Bourdin $S$, Lesaunier $F$, Benassi $T$, Lemanski C, Geoffrois L, Lusinchi $A$, Verrelle $P$, Bardet $E$, Julieron $M$, Wibault $P$, Luboinski $M$, Benhamou $E$. Phase III randomized trial of very accelerated radiation therapy compared with conventional radiation therapy in squamous cell head and neck cancer: a GORTEC trial. J Clin Oncol 2006;24:2873-8.

4. Lapeyre M, Charra-Brunaud C, Kaminsky MC, Geoffrois L, Dolivet G, Toussaint B, Maire F, Pourel N, Simon M, Marchal C, Bey P. Management of mucositis following radiotherapy for head and neck cancers. Cancer Radiother 2001;5 Suppl 1:121s-30s.

5. Sonis ST, Elting LS, Keefe D, Peterson DE, Schubert M, Hauer-Jensen M, Bekele BN, Raber-Durlacher J, Donnelly JP, Rubenstein EB. Perspectives on cancer therapy-induced mucosal injury: pathogenesis, measurement, epidemiology, and consequences for patients. Cancer 2004;100(9 Suppl):1995-2025.

6. Scully C, Sonis S, Diz PD. Oral mucositis. Oral Dis 2006;12:22941.

7. Bensadoun RJ, Le Page F, Darcourt V, Bensadoun F, Ciais G, Rostom YA, Poissonnet G, Dassonville 0, Demard F. MASCC/ISO0 mucositis group.Radiation-induced mucositis of the aerodigestive tract: prevention and treatment. MASCC/ISO0 mucositis group's recommendations. Bull Cancer 2006;93:201-11.

8. Rosenthal DI, Trotti A. Strategies for managing radiationinduced mucositis in head and neck cancer. Semin Radiat Oncol 2009;19:29-34.

9. Hensley ML, Hagerty KL, Kewalramani T, Green DM, Meropol NJ, Wasserman TH, Cohen GI, Emami B, Gradishar WJ, Mitchell RB, Thigpen JT, Trotti A 3rd, von Hoff D, Schuchter LM. American Society of clinical Oncology 2008 clinical practice guideline update: use of chemotherapy and radiation therapy protectants. J Clin Oncol 2009;27:127-45. 
10. Cowen D, Tardieu C, Schubert M, Peterson D, Resbeut M, Faucher $C$, Franquin JC. Low energy Helium-Neon laser in the prevention of oral mucositis in patients undergoing bone marrow transplant: results of a double blind randomized trial. Int J Radiat Oncol Biol Phys 1997;38:697-703.

11. Antunes HS, de Azevedo AM, da Silva Bouzas LF, Adão CA, Pinheiro CT, Mayhe R, Pinheiro LH, Azevedo R, D'Aiuto de Matos V, Rodrigues PC, Small IA, Zangaro RA, Ferreira CG. Lowpower laser in the prevention of induced oral mucositis in bone marrow transplantation patients: a randomized trial. Blood 2007;109:2250-5.

12. Schubert MM, Eduardo FP, Guthrie KA, Franquin JC, Bensadoun RJ, Migliorati CA, Lloid CM, Eduardo CP, Walter NF, Marques MM, Hamdi M. A phase III randomized doubleblind placebocontrolled clinical trial to determine the efficacy of low level laser therapy for the prevention of oral mucositis in patients undergoing hematopoietic cell transplantation. Support Care Cancer 2007;15:1145-154.

13. Bensadoun RJ, Franquin JC, Ciais G, Darcourt V, Schubert MM, Viot M, Dejou J, Tardieu C, Benezery K, Nguyen TD, Laudoyer Y, Dassonville 0, Poissonnet G, Vallicioni J, Thyss A, Hamdi M, Chauvel $\mathrm{P}$, Demard F. Low-energy He/Ne laser in the prevention of radiation-induced mucositis. A multicenter phase III randomized study in patients with head and neck cancer. Support Care Cancer 1999.

14. Arora H, Pai KM, Maiya A, Vidyasagar MS, Rajeev Efficacy of He-Ne Laser in the prevention and treatment of radiotherapyinduced oral mucositis in oral cancer patients. Oral Surg Oral Med Oral Pathol Oral Radiol Endod 2008;105:180-6.

15. Simões A, Eduardo FP, Luiz AC, Campos L, Sá PH, Cristófaro M, Marques MM, Eduardo CP. Laser phototherapy as topical prophylaxis against head and neck cancer radiotherapy-induced oral mucositis: comparison between low and high/low power lasers. Lasers Surg Med 2009;41:264-70.

16. Zanin T, Zanin F, Carvalhosa AA, de Souza Castro PH, Pacheco MT, Zanin IC, Junior AB. Use of $660-\mathrm{nm}$ diode laser in the prevention and treatment of human oral mucositis induced by radiotherapy and chemotherapy. Photomed Laser Surg 2009 Sep 21. [Epub ahead of print].

17. Eduardo FP, Bezinelli L, Luiz AC, Correa L, Vogel C, Eduardo CP. Severity of oral mucositis in patients undergoing hematopoietic cell transplantation phototherapy protocol: a survey of $30 \mathrm{pa}-$ tients. Photomed Laser Surg 2008;26:1-8.

18. Lopes C, MAS JRI, Zângaro RA. Low level laser therapy in the prevention of radiotherapy-induced xerostomia and oral mucositis. Radiol Bras 2006;39:131-136.

19. Nes AG, Posso MBS. Patients with moderate chemotherapyinduced mucositis: pain therapy using low intensity lasers. Int Nurs Rev 2005;52:68-72.
20. Moriyama Y, Moriyama EH, Blackmore K, Akens M, Lilge L. In vivo study of the inflammatory modulating effects of low-level laser therapy on iNOS expression using bioluminescence imaging. Photochem Photobiol 2005;81:1351-5.

21. Sonis ST. The pathobiology of mucositis. Nat Rev Cancer 2004:4:277-84

22. Mafra de Lima F, Costa MS, Albertini R, Silva JA Jr, Aimbire F. Low level laser therapy (LLLT): attenuation of cholinergic hyperreactivity, beta(2)-adrenergic hyporesponsiveness and TNFalpha mRNA expression in rat bronchi segments in $\mathrm{E}$. coli lipopolysaccharide-induced airway inflammation by a NF-kappaB dependent mechanism. Lasers Surg Med 2009;41:68-74.

23. Correa F, Lopes Martins RA, Correa JC, Iversen VV, Joenson $\mathrm{J}$, Bjordal JM. Low-level laser therapy (GaAs lambda $=$ $904 \mathrm{~nm}$ ) reduces inflammatory cell migration in mice with lipopolysaccharide-induced peritonitis. Photomed Laser Surg 2007;25:245-9.

24. Lim W, Lee S, Kim I, Chung M, Kim M, Lim H, Park J, Kim 0, Choi $\mathrm{H}$. The anti-inflammatory mechanism of $635 \mathrm{~nm}$ light-emittingdiode irradiation compared with existing COX inhibitors. Lasers Surg Med 2007;39:614-21.

25. Mizutani K, Musya Y, Wakae K, Kobayashi T, Tobe M, Taira K, Harada T. A clinical study on serum prostaglandin E2 with lowlevel laser therapy. Photomed Laser Surg 2004;22:537-9.

26. Hagiwara S, Iwasaka H, Okuda K, Noguchi T. GaAlAs (830 nm) low-level laser enhances peripheral endogenous opioid analgesia in rats. Lasers Surg Med 2007;39:797-802.

27. Gur A, Karakoc M, Cevik R, Nas K, Sarac AJ, Karakoc M. Efficacy of low power laser therapy and exercise on pain and functions in chronic low back pain. Lasers Surg Med 2003;32:233-828.

28. Chow RT, David MA, Armati PJ. 830 nm laser irradiation induces varicosity formation, reduces mitochondrial membrane potential and blocks fast axonal flow in small and medium diameter rat dorsal root ganglion neurons: Implications for the analgesic effects of $830 \mathrm{~nm}$ laser. J Peripher Nerv Syst 2007;12:28-39.

29. Almeida AL, Esper LA, Sbrana MC, Ribeiro IW, Kaizer RO. Utilization of low-intensity laser during healing of free gingival grafts. Photomed Laser Surg 2009;27:561-4.

30. França CM, França CM, Núñez SC, Prates RA, Noborikawa E, Faria $M R$, Ribeiro MS. Low-intensity red laser on the prevention and treatment of induced-oral mucositis in hamsters. Photobiol B 2009;94:25-31.

31. Almeida-Lopes L, Rigau J, Zangaro RA, Guidugli-Neto J, Jaeger MM. Comparison of the low level laser therapy effects on cultured human gingival fibroblasts proliferation using different irradiance and same fluence. Lasers Surg Med 2001;29:179-84.

32. Pereira AN, Eduardo Cde P, Matson E, Marques MM. Effect of lowpower laser irradiation on cell growth and procollagen synthesis of cultured fibroblasts. Lasers Surg Med 2002;31:263-7. 